\title{
CARACTERIZAÇÃO DA VIOLÊNCIA CONTRA MULHERES IDOSAS NO MUNICÍPIO DE VITÓRIA DA CONQUISTA/BA
}

\author{
Mércia Mendonça*, Luciana Araújo dos Reis** \\ Autor correspondente: Luciana Araújo dos Reis - lucianareis@fainor.com.br \\ * Discente do curso de Enfermagem do $10^{\circ}$ semestre da Faculdade Independente do Nordeste. \\ ** Docente da disciplina TCC II da Faculdade Independente do Nordeste, com Doutorado em Ciências da Saúde pela Universidade Fede- \\ ral do Rio Grande do Norte e Pós-Doutorado em Saúde Coletiva pelo Instituto de Saúde Coletiva da Universidade Federal da Bahia.
}

\section{Resumo}

(Objetivo) caracterizar a violência contra mulheres idosas no município de Vitória da Conquista/BA. (Metodologia) trata-se de uma pesquisa de ordem quantitativa exploratória, realizada na Delegacia Especializada de Atendimento a Mulher/DEAM de Vitória da Conquista/BA no período de janeiro de 2012 a outubro de 2014, sendo incluído como amostra do estudo todas as mulheres idosas vítimas de violência no tempo definido para a coleta de dados do projeto. (Resultados) constatou-se no presente estudo que a média de idade das idosas avaliadas foi $69,45( \pm 7,98)$ anos e que houve uma maior frequência de idosas com estado civil de casada (34,0\%), com nível de escolaridade referente a não alfabetizada $(44,3 \%)$ e com renda de or salário mínimo $(65,1 \%)$. Em relação ao tipo de violência verificou-se que houve uma maior distribuição de violência psicológica $(50,0 \%)$ e física $(25,5 \%)$. (Conclusão) sendo mais comuns as agressões realizadas por filhos $(35,8 \%)$ e noras $(34,9 \%)$. Com base nos resultados verificou-se que os principais tipos de violência sofridos pelos idosos foram a psicológica e física, sendo os filhos e noras os principais agressores.

Palavras-chave: Idoso; Violência; Maus-tratos.

\section{CHARACTERIZATION OF VIOLENCE AGAINST ELDERLY WOMEN IN THE CITY OF VICTORY OF CONQUEST/BA}

\begin{abstract}
(Objective) characterize violence against older women in Vitória da Conquista / BA. (Methodology) it is a survey of exploratory quantitative, held at the Police Specialized Care for Women/DEAM of Vitória da Conquista / BA from January 2012 to October 2014, being included as study sample all women elderly
\end{abstract}

- Artigo submetido para avaliação em 14/11/2O14 e aceito para publicação em 14/O1/2O16 • 
victims of violence in the time set for the data collection project. (Results) it was found in this study that the average age of elderly women was $69.45( \pm 7.98)$ years and there was a higher frequency in elderly women with marital status of married (34.0\%), with level of the illiterate (44.3\%) and 01 with incomes of minimum wage (65.1\%) regarding education. Regarding the type of violence it was found that there was a greater distribution of psychological (50.0\%) and physical (25.5\%). (Conclusion) being the most common attacks performed by children $(35.8 \%)$ and daughters $(34.9 \%)$. Based on the results it was found that the main types of violence suffered by the elderly was psychological and physical, the sons and daughters being the main offenders.

Keywords: Elderly; Violence; III-treatment.

\section{INTRODUÇÃO}

No Brasil, o número de idosos passou de três miIhões, em 1960, para sete milhões em 1975 e 14 milhões em 2002. Estima-se que alcançará $34 \mathrm{mi}-$ Ihões em 2020, levando o país ao sexto lugar no ranking mundial de países com maior número de pessoas nessa faixa etária. ${ }^{(1)}$

Esse crescimento traz a consciência da existência da velhice como questão social, questão esta que pede grande atenção, pois esta diretamente relacionada com a crise de identidade, a mudança de papeis, a aposentadoria, as perdas diversas, as violências e a diminuição dos contatos sociais. ${ }^{(2)}$ Neste sentido, o Brasil tem ampliado políticas para a criação de serviços voltados para os idosos, como os centros de referência multiprofissional, as casas de abrigo e as instituições próprias para denúncias de violência contra os idosos.

A violência contra o idoso é um fenômeno que não se restringe a realidade de um país, de uma cidade ou localidade, mas, trata-se de um fenômeno complexo, que atinge tanto os países desenvolvidos, como os países subdesenvolvidos. Trata-se de um problema social, político e de saúde, pois afeta a integridade corporal e o estado psíquico e emocional da vítima, acarretando sérias e graves consequências para o seu pleno e integral desenvolvimento, comprometendo- Ihe o exercício da cidadania e dos direitos humanos. ${ }^{(3)}$
A violência é o uso intencional da força física ou do poder, real ou ameaça, contra si próprio ou contra outra pessoa, grupo ou comunidade, que resulte ou possibilite resultar em lesão, morte, dano psicológico, deficiência de desenvolvimento ou privação. (4) A Assembleia Mundial de Saúde declarou a violência como um dos problemas de saúde publica, e convocou à OMS para desenvolver uma tipologia da violência, bem como os vínculos entre elas.

Quando se fala em violência contra o idoso, pensa-se imediatamente na violência física, mas esta não é a única, pois há inúmeras formas de violência. Estudos demonstram que a maior parte das violências sofridas pelos mesmos ocorre nos lares, sendo os principais agressores os filhos, as noras, os genros e os cônjuges, havendo uma forte associação com o uso de álcool e drogas, relação de dependência financeira entre pais e filhos, história de violência na família, sofrimento mental e psiquiátrico, entre outros. ${ }^{(5)}$

Para a sociedade em geral, as produções cientificas sobre este tema, constituem-se para entender melhor e conhecer sobre abusos e maus-tratos contra mulheres idosas, assunto ainda negligenciando e esquecido por todos. Tomar conhecimento do que se acontece com as mulheres idosas e o que tem se feito para combater a violência, é importante para que os órgãos públicos busquem soluções imediatas para combater esse tipo de dano 
à mulher idosa, por essa razão justifica-se este estudo. Nesta perspectiva, o presente estudo tem por objetivo: caracterizar a violência contra mulheres idosas no município de Vitória da Conquista/BA.

\section{METODOLOGIA}

Trata-se de uma pesquisa exploratória descritiva com abordagem quantitativa. A escolha por este tipo de investigação deve-se ao fato de que esta permite ampliar o conhecimento em relação ao objeto, possibilitando trazer indicadores e tendências com segurança e fazer inferências sobre o fenômeno estudado.

A pesquisa quantitativa envolve a coleta sistemática de dados, com ênfase na objetividade e sua análise se dá normalmente por meio de procedimento estatístico6. Neste método, a repetição das informações é importante para a segurança quanto à inferência, uma vez que busca a precisão matemática e a descrição numérica dos dados, além de permitir maior generalização de resultados e comparações entre populações diferentes. ${ }^{(6)}$

A pesquisa foi realizada na Delegacia Especializada de Atendimento a Mulher/DEAM de Vitória da Conquista/BA no período de janeiro de 2012 a outubro de 2014, sendo incluídas como amostra do estudo todas as mulheres idosas vítimas de violência no tempo definido para a coleta de dados da pesquisa.

A DEAM compõe a estrutura da Polícia Civil, devendo realizar ações de prevenção, apuração, investigação e enquadramento legal. Entre as ações, cabe citar: registro de Boletim de Ocorrência e do termo de representação, solicitação ao juiz das medidas protetivas de urgência nos casos de violência doméstica contra as mulheres.
Como instrumento para coleta de dados foi utilizado um questionário contendo questões referentes a informações sociodemográficas (idade, nível de escolaridade, renda e estado civil) e sobre violência (tipo de violência e agressor).

A DEAM possui um banco de dados no qual são registradas as ocorrências, contendo informações sociodemográficas e sobre a violência. Para a realização do presente estudo foram utilizados apenas os registros das ocorrências envolvendo mulheres idosas no período de janeiro de 2012 a outubro de 2014.

Após a aprovação do projeto de pesquisa pelo Comitê de Ética e Pesquisa da Faculdade Independente do Nordeste CEP/FAINOR, foi encaminhado um ofício a referida delegacia solicitando autorização para a coleta de dados. Os dados foram coletados nos prontuários de ocorrência pela própria autora da pesquisa.

Os dados foram analisados de maneira descritiva por meio do Programa estatístico SPSS versão 20.00. O projeto de pesquisa foi submetido e aprovado pelo Comitê de Ética da Faculdade Independente do Nordeste/FAINOR (Parecer $n^{\circ}$ 16506513.3.0000.5578). Para participação na pesquisa cada idoso assinou um Termo de Consentimento Livre e Esclarecido.

\section{RESULTADOS E DISCUSSÕES}

Constatou-se no presente estudo que a média de idade das idosas avaliadas foi $69,45( \pm 7,98)$ anos e que houve uma maior frequência de idosas casadas $(34,0 \%)$, não alfabetizada $(44,3 \%)$ e com renda de or salário mínimo $(65,1 \%)$. 


\begin{tabular}{lcc}
\hline & $N$ & $\%$ \\
\hline Estado Civil & 25 & 23,6 \\
Solteira & 36 & 34,0 \\
Casada & 11 & 10,4 \\
Divorciada & 29 & 27,4 \\
Viúva & 5 & 4,7 \\
Outros & & \\
Nível de Escolaridade & 47 & 44,3 \\
Não alfabetizada & 28 & 26,4 \\
$1^{\circ}$ Grau Incompleto & 16 & 15,1 \\
$1^{\circ}$ Grau completo & 3 & 2,8 \\
$2^{\circ}$ Grau Incompleto & 9 & 8,5 \\
$2^{\circ}$ Grau Completo & 1 & 0,9 \\
Nível Superior incompleto & 2 & 1,9 \\
Nível Superior completo & & \\
Renda & 25 & 23,6 \\
Menos de 1 salário mínimo & 69 & 65,1 \\
Ol salário mínimo & 10 & 9,4 \\
O2 salários mínimos & 2 & 1,9 \\
O3 ou mais salários mínimos & 106 & 100,0 \\
\hline Total & &
\end{tabular}

A média de idade encontrada no presente estudo é semelhante aos resultados encontrados em pesquisa realizada por Mascarenhas et al. ${ }^{(2)}$ sobre violência contra a pessoa idosa no qual a média de idade dos idosos do sexo feminino vítimas de violência foi de 71,1 anos ( $\pm 8,8$ anos), variando de 60 a 109 anos. Aproximadamente metade das idosas tinha de 60 a 69 anos de idade (50,2\%).

Em estudo realizado por Duque et al.(4) sobre Violência contra idosos no ambiente doméstico as vítimas que sofreram violência e que nunca estudaram (analfabetas) foram as que mais sofreram violência $(26,15 \%)$, assim como as que não tinham companheiro $(21,71 \%)$. Ressalte-se ainda que foram mais vitimizados os idosos que recebiam até um salário mínimo $(22,86 \%)$ e os que contribuíam para o sustento da casa $(20,88 \%)$. Estes dados são semelhantes ao do presente estudo em relação ao nível de escolaridade e a renda, pois a maioria das idosas vítimas de violência era do sexo feminino e com renda referente a 1 salário mínimo. Porém, são contraditórios aos resultados do presente estudo em relação ao estado civil, uma vez que, nesta pesquisa as idosas que mais sofreram violência foram as casadas.

Em relação ao tipo de violência verificou-se que houve uma maior distribuição de violência psicológica $(50,0 \%)$ e física $(25,5 \%)$. Sendo mais comuns as agressões realizadas por filhos $(35,8 \%)$ e noras $(34,9 \%)$. 
Tabela 2 - Distribuição do tipo de violência e agressor. Vitória da Conquista/BA, 2014.

\begin{tabular}{lcc}
\hline & $\mathrm{N}$ & $\%$ \\
\hline Tipo de Violência & 27 & \\
$\quad$ Violência física & 2 & 25,5 \\
Violência sexual & 53 & 1,9 \\
Violência psicológica & 14 & 50,0 \\
Violência matrimonial & 10 & 13,2 \\
Outros & & 9,4 \\
Agressor & 18 & \\
Companheiro & 38 & 17,0 \\
Filho (a) & 5 & 35,8 \\
Genro & 37 & 4,7 \\
Nora & 8 & 34,9 \\
$\quad$ Netos & 106 & 7,5 \\
\hline Total & & 100,0 \\
\hline
\end{tabular}

Conforme a Tabela 2 o tipo de violência que apresentou uma maior frequência foram a violência psicológica e física, sendo estes dados corroborados por pesquisa realizada por Mascarenhas et al.(2) na qual o tipo de violência mais relatados em seu trabalho foram: abuso físico $(67,7 \%)$, violência psicológica $(29,1 \%)$ e negligência $(27,8 \%)$. Em menor proporção, foram referidos casos de violência financeira $(7,9 \%)$, abuso sexual $(3,7 \%)$ e tortura $(3,3 \%) \cdot(7)$

Em estudo realizado por Caldas et al. ${ }^{(8)}$ no qual foi realizado um levantamento em uma população cadastrada no Programa Médico da Família, em Niterói (RJ), com o objetivo de estimar a prevalência de violência Física em idosas foi estimado que $10,1 \%$ das idosas entrevistadas sofreram violência no ano anterior à entrevista. Em publicação mais recente, Apratto Júnior, ${ }^{(1)}$ com a população de mesmas características, estimou a prevalência da violência psicológica em 43,2\%.

Segundo os dados da Tabela 2 nota-se que sobre o agressor o filho e a nora foram os que mais prevaleceram como causadores da agressão contra as mulheres idosas. Conforme a literatura sobre violência contra idosos os maiores agressores no contexto doméstico e familiar são os filhos das idosas, seguidos do companheiro ou genros/noras. ${ }^{(9)}$

$\mathrm{Na}$ distribuição dos números de ocorrências de violência contra idosas observou-se que no ano de 2012 houve uma redução dos números de ocorrências (30 ocorrências) quando comparados com os números de ocorrências do ano de 2013 (44 ocorrências).

Figura 1 - Distribuição dos números de ocorrências de violência contra idosas. Vitória da Conquista/BA, 2014

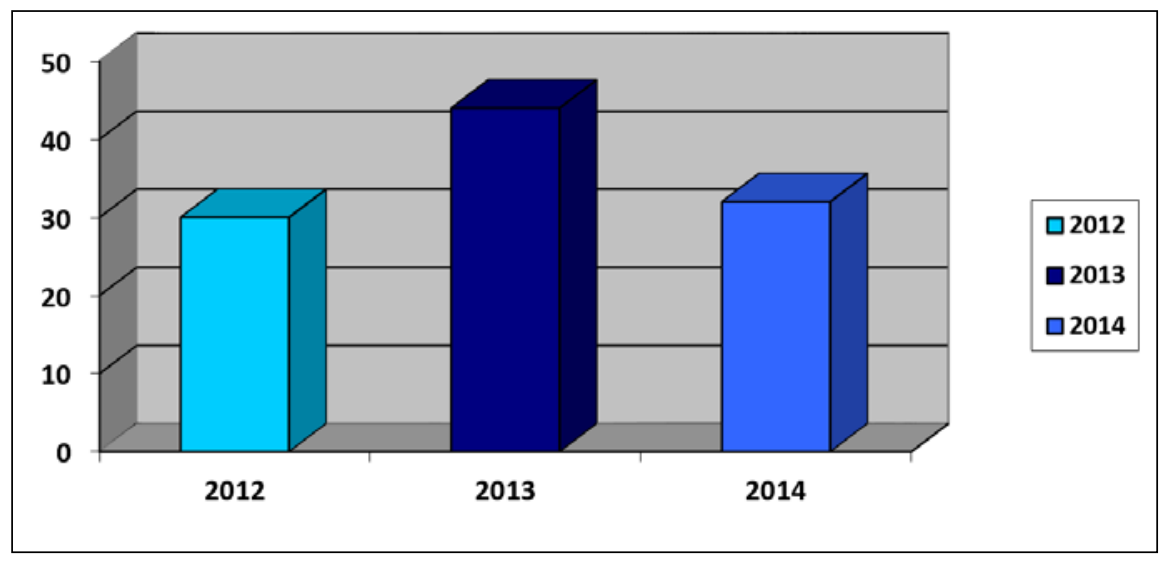

- Revista Enfermagem Contemporânea. 2015 Jul./Dez;4(2):129-136 • 
Este aumento no número de ocorrências do número de 2013 em relação ao ano de 2012 pode ser justificado pela maior divulgação do Estatuto do Idoso junto a população idosa, o que contribuiu para uma maior sensibilização desta população sobre os seus direitos e sobre as formas de reivindicá-los. Enquanto que o número menor de casos no ano de 2014 quando comparado ao ano de 2013 , pode ser justificado pelo fato que no ano de 2013 foram abordadas as ocorrências de janeiro a dezembro, enquanto que no ano de 2014 foi de janeiro a outubro.

Em pesquisa realizada por Carneiro et al.(1) sobre a Estatística da violência contra idosos em Guarapuava (PR) ficou evidente que: no período de janeiro de 2007 a julho de 2011 foram registradas 138 denúncias de violência contra a pessoa idosa atra- vés do Disque Idoso em Guarapuava (PR). Segundo os dados, no primeiro ano de registros, 2007, o número de denúncias foram apenas 6 , enquanto nos anos seguintes a média foi de 33 denúncias anuais, representando significativo aumento do uso do serviço Disque Idoso. Observa-se que a média semestral de denúncias entre 2007 e 2010 foi de 15,2 , enquanto no primeiro semestre de 2011 foram recebidas 22 denúncias, o que indica uma tendência de maior uso do serviço Disque Idoso pela população de Guarapuava.

Quanto ao tipo de violência segundo o ano de ocorrência constatou-se que no ano de 2012 houve uma maior distribuição de violência física (13 casos), no ano de 2013 foi mais frequente a violência psicológica (24 casos) e, em 2014 foi mais comum a violência psicológica.

Figura 2 - Distribuição dos tipos de violência segundo o ano de ocorrência. Vitória da Conquista/BA, 2014

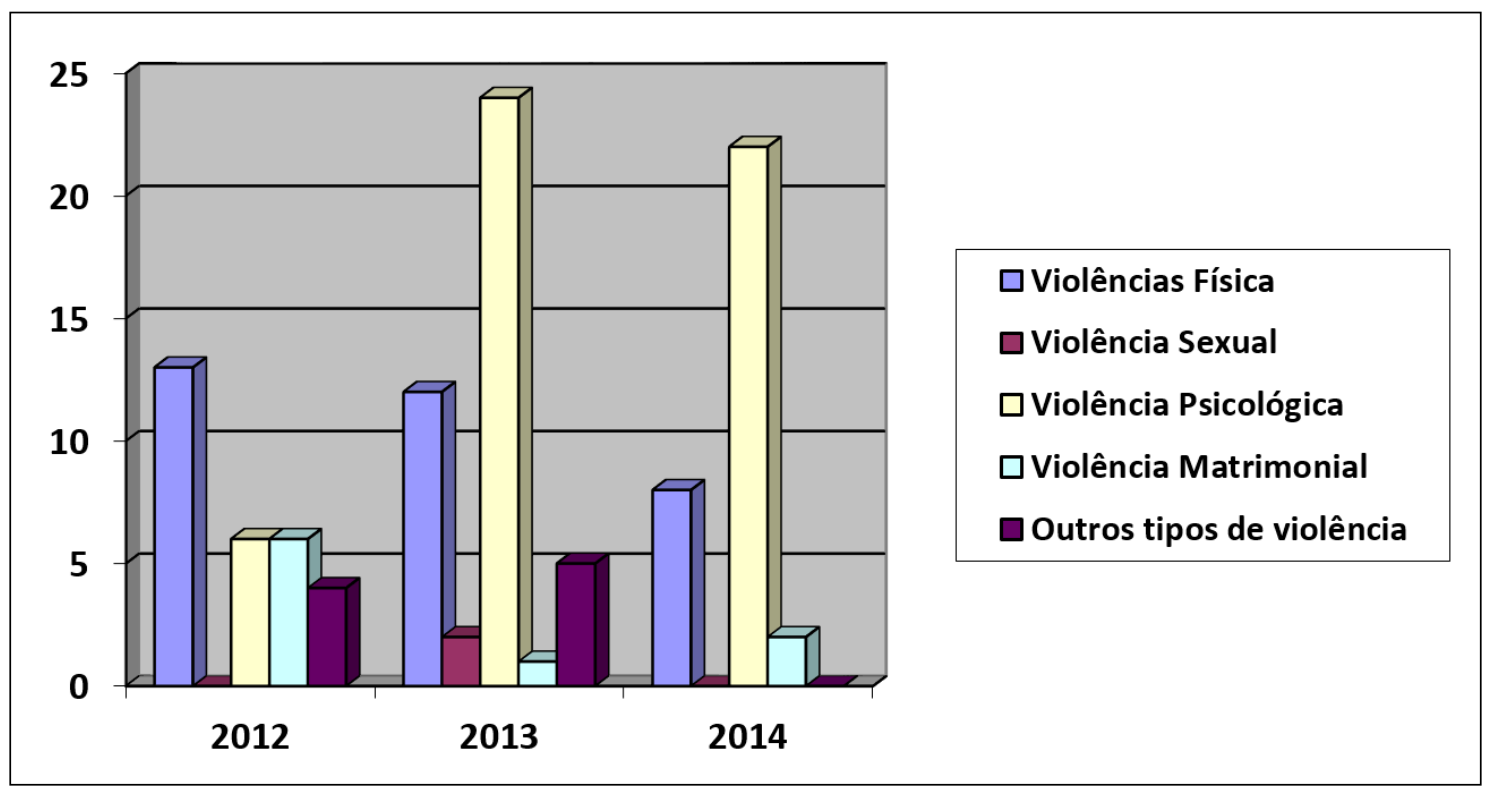

Em estudo realizado por Mascarenhas et al. ${ }^{(2)}$ sobre os tipos de violência mais frequentes ficou evidente que o abuso físico predominou $(67,7 \%)$, violência psicológica $(29,1 \%)$ e negligência (27,8\%). Em menor proporção, foram referidos casos de violência financeira $(7,9 \%)$, abuso sexual $(3,7 \%)$ e tortura $(3,3 \%)$.
Observa-se que no estudo citado acima que os tipos de violência mais frequentes estão compatíveis com o resultado da deste estudo, concluindo que a violência física e psicológica está predominante nos dois estudos.

Pesquisa realizada por Duque et al.(4) aponta que sobre os tipos de violência contra idosos hou- 
ve predominância da agressão física com 28 casos, financeira 25 casos e psicológica 23 casos e, além disso, o registro de duas denúncias de violência sexual.

Em revisão sistemática sobre a prevalência de violência física na terceira idade, Espíndola e Blay" encontraram estudos conduzidos em diversas regiões do mundo, principalmente nos Estados Unidos e na Europa, que apontavam a prevalência de abuso físico na população idosa variando de 1,2\% (Holanda) a 18\% (Finlândia). Nota-se que a violência física é maior na Finlândia.

Assim como Apratto Júnior ${ }^{(1)}$ demonstrou que, independentemente do tipo de violência sofrida psicológica ou física - as prevalências eram maiores entre os idosos que coabitavam em ambiente com um número maior de residentes, $61,5 \%$ e $19,2 \%$, respectivamente.

Figura 3 - Distribuição do tipo de agressor segundo o ano de ocorrência. Vitória da Conquista/BA, 2014

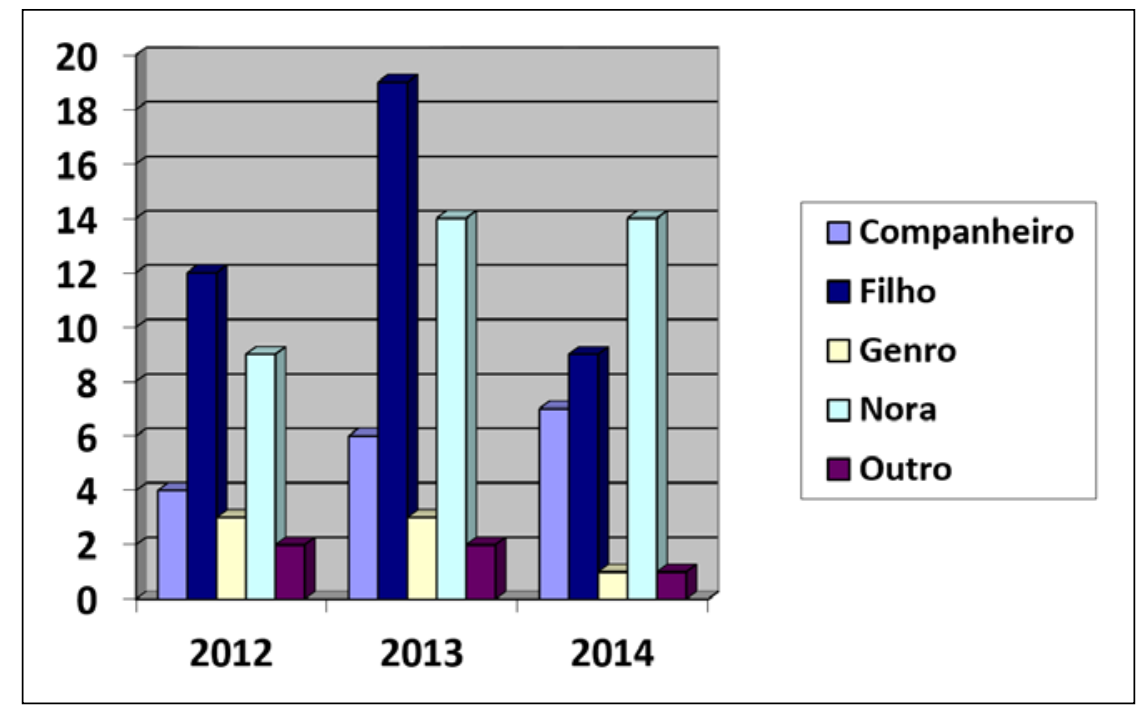

Quanto ao tipo de agressor em relação ao ano de ocorrência da violência, observou-se que nos anos de 2012 e 2013 os principais agressores foram os filhos, e em 2014 as noras.

Segundo estudo realizado por Sousa et al. ${ }^{(12)}$ sobre os tipos de agressores com idosos, os maiores agressores no contexto doméstico são os filhos dos idosos, seguidos do companheiro ou genros/ noras. Somado a isso, teria que se considerar especificidades deste companheiro, uma vez que, como já destacado, no surgimento da violência os fatores parecem estar inter-relacionados.

Utilizando-se uma categorização diferente desta pesquisa, Apratto Júnior apontou que as vítimas mais frequentes estavam no grupo dos casados quando comparados os viúvos, tanto para a violência psicológica quanto para a violência física grave e total. Gaioli e Rodrigues ${ }^{(9)}$ apontaram que as maiores vítimas estavam entre os casados ou amasiados $(46,4 \%)$. Moraes et al. ${ }^{(13)}$ registraram que foram mais vitimizados os idosos que tinham companheiro, embora a classificação esteja bem diferenciada da presente pesquisa.

\section{CONSIDERAÇÕES FINAIS}

Constatou-se no presente estudo que houve uma maior frequência de idosas com estado civil de casada, com nível de escolaridade referente a não alfabetizada e com renda de or salário mínimo. Em relação ao tipo de violência verificou-se que houve uma maior distribuição de violência psicológica e 
física. Sendo mais comuns as agressões realizadas por filhos e noras.

\section{REFERÊNCIAS}

1. Apratto Junior PC. A violência doméstica contra idosos nas áreas de abrangência do Programa Saúde da Família de Niterói (RJ, Brasil). Ciênc. saúde coletiva. 2010;15(6):2983-2995.

2. Mascarenhas MDM, Andrade ACSS, Neves MCA, Pedrosa GAA, Silva AMM, Malta CD. Violência contra a pessoa idosa: análise das notificações realizadas no setor saúde - Brasil, 2010. Ciênc. saúde coletiva. 2012;17(9):23312341, 2012.

3. Ilha MM, Leal SMC, Soares JSF. Mulheres internadas por agressão em um hospital de pronto socorro: (in)visibilidade da violência. Rev. gaúch. enferm. 2010;31(2): 328-34.

4. Duque MA, Leal CCM, Marques OPA, Eskinazi VMF, Duque MA. Violência contra idosos no ambiente doméstico: prevalência e fatores associados (Recife/PE). Ciênc. saúde coletiva. 2O12;17(8).

5. Wanderbroocke, AC, More C. Significados de violência familiar para idosos no contexto da atenção primária. Psicol. Teor. Pesqui. 2012;28(4):435-442.

6. Richardson RJ. et al. Pesquisa Social: métodos e técnicas. $3^{a}$ ed. São Paulo: Atlas; 2008.
7. Shimbo AY. O reconhecimento pela equipe da estratégia saúde da família da violência intrafamiliar contra idosos [dissertação]. Curitiba: Universidade Federal do Paraná; 2008.

8. Caldas SL, Fortaleza RK, Silva CVP, Brito OML, Chein CBM, Vasconcelos BC. Violência contra a mulher idosa: vozes silenciadas. Geriatria \& Gerontologia. 2009;2(4):167-175.

9. Gaioli CCLO, Rodrigues RAP. Ocorrência de maus-tratos em idosos no domicílio. Rev. latinoam. Enferm. 2008;16(3).

1O. Carneiro OS, Schimanski E. Violência contra a pessoa idosa: uma questão de gênero? Publicatio UEPG: Ciências Sociais Aplicadas. 2014;22(2):167- 177.

11. Espíndola CR; Blay SL. Prevalência de maus tratos na terceira idade: revisão sistemática. Rev. saúde pública. 2007;41(2):301-306.

12. Souza ER, Ribeiro AP, Atie S, Souza AC, Marques CC. Rede de proteção aos idosos do Rio de Janeiro: um direito a ser conquistado. Ciênc. saúde coletiva. 2008;13(4):1153-1163.

13. Moraes $\mathrm{CL}$, Apratto Júnior PC, Reichenheim ME. Rompendo o silêncio e suas barreiras: um inquérito domiciliar sobre a violência doméstica contra idosos em área de abrangência do Programa Médico da Família de Niterói, Rio de Janeiro, Brasil. Cad. saúde pública. 2008;24(10):2289-2300. 